\title{
Multisemigroups with multiplicities and complete ordered semi-rings
}

\author{
Love Forsberg $^{1}$ (D)
}

Received: 24 February 2016 / Accepted: 5 October 2016 / Published online: 4 November 2016 (C) The Author(s) 2016. This article is published with open access at Springerlink.com

\begin{abstract}
Motivated by the appearance of multisemigroups in the study of additive 2categories, we define and investigate the notion of a multisemigroup with multiplicities. This notion seems to be well suited for applications in higher representation theory.
\end{abstract}

Keywords Semigroup $\cdot$ Multisemigroup $\cdot 2$-category $\cdot$ Representation theory

Mathematics Subject Classification 18D05 - 20M50

\section{Introduction}

Abstract 2-representation theory originates from the papers (Bernstein et al. 1999; Khovanov 2000; Chuang and Rouquier 2008) and is nowadays formulated as the study of 2-representations of additive $\mathbb{k}$-linear 2-categories, where $\mathbb{k}$ is the base field, see e.g. Mazorchuk (2012) for details. Various aspects of general 2-representation theory of abstract additive $\mathbb{k}$-linear 2-categories were studied in the series (Mazorchuk and Miemietz 2011, 2014, 2016a, b) of papers by Mazorchuk and Miemietz. An important role in this study is played by the so-called multisemigroup of an additive $\mathbb{k}$-linear 2category which was originally introduced in Mazorchuk and Miemietz (2016b).

Recall that a multisemigroup is a set $S$ endowed with a multioperation, that is a map $*: S \times S \rightarrow 2^{S}$ which satisfies the following associativity axiom:

$$
\bigcup_{s \in a * b} s * c=\bigcup_{t \in b * c} a * t
$$

\footnotetext{
$\bowtie$ Love Forsberg

love.forsberg@math.uu.se

1 Department of Mathematics, Uppsala University, Box. 480, 75106 Uppsala, Sweden
} 
for all $a, b, c \in S$ (see Kudryavtseva and Mazorchuk 2015 for more details and examples). This is the precise notion of associativity that makes $2^{S}$ into a semigroup with the usual notion of associativity. The original observation in Mazorchuk and Miemietz (2016b) is that the set $\mathcal{S}$ of isomorphism classes of indecomposable 1-morphisms in an additive $\mathbb{k}$-linear 2 -category $\mathscr{C}$ has the natural structure of a multisemigroup, given as follows: for two indecomposable (which in this setting is well-defined) 1-morphisms $\mathrm{F}$ and $\mathrm{G}$, we have

$$
[\mathrm{F}] *[\mathrm{G}]=\{[\mathrm{H}]: \mathrm{H} \text { is isomorphic to a direct summand of } \mathrm{F} \circ \mathrm{G}\},
$$

where $[\mathrm{F}]$ stands for the isomorphism class of $\mathrm{F}$ and o denotes composition in $\mathscr{C}$. We refer the reader to Mazorchuk and Miemietz (2016b) for details. The combinatorics of this multisemigroup reflects and encodes various structural properties of the underlying additive $\mathbb{k}$-linear 2-category and controls major parts of the 2-representation theory of the latter, see Mazorchuk and Miemietz (2011, 2014, 2016a, b) for details.

However, this notion of a multisemigroup of an additive $\mathbb{k}$-linear 2-category has one disadvantage: it seems to forget too much information. In more details, it only records information about direct summands appearing in the composition $\mathrm{F} \circ \mathrm{G}$, however, it forgets information about the multiplicities with which these direct summands appear. As as result, the multisemigroup of an additive $\mathbb{k}$-linear 2-category can not be directly applied to the study of the split Grothendieck category of $\mathscr{C}$ and linear representations of the latter.

It is quite clear how one can amend the situation: one has to define a weaker notion than a multisemigroup which should keep track of multiplicities in question. This naturally leads to the notion of multisemigroups with multiplicities, or multimultisemigroups which is the object of the study in this paper (the idea of such an object is mentioned in [Mazorchuk and Miemietz (2016b), Remark 8] without any details). Although the definition is rather obvious under a natural finiteness assumption, the setup in full generality has some catches and thus requires some work. The main aim of the present paper is to analyze this situation and to propose a "correct" definition of a multi-multisemigroup. The main value of the paper lies not in the difficulty of the results presented but rather in the thorough analysis of the situation which explores various connections of the theory we initiate. Our approach utilizes the algebraic theory of complete semirings.

The paper is organized as follows: in Sect. 2 we outline in more detail the motivation for this study coming from higher representation theory. In Sect. 3 we collect all notions and tools necessary to define our main object: multi-multisemigroups, or, how we also call them, multisemigroups with multiplicities bounded by some cardinal. Section 4 ties back to the original motivation and is devoted to the analysis of multisemigroups with multiplicities appearing in higher representation theory. In Sect. 5 we give some explicit examples. In Sect. 6 we discuss multi-multisemigroups for different sets of multiplicities and connection to twisted semigroup algebras. Finally, in Sect. 7, we describe multi-multisemigroups as algebras over complete semirings. 


\section{Motivation from 2-representation theory}

\subsection{2-categories}

For details on 2-categories we refer the reader to Leinster (1998); Mazorchuk (2012). A 2-category is a category enriched by small categories. Explicitely this means that $\mathscr{C}$ consists of the following data:

- Objects i,j,

- 1-morphisms $f, g \mathrm{i} \rightarrow \mathrm{j}$ between objects, including an identity 1-morphism $1_{\mathrm{i}}$ : $\mathrm{i} \rightarrow \mathrm{i}$ for each object $\mathrm{i}$,

- 2-morphisms $\alpha, \beta: f \rightarrow g$ between 1-morphisms, including an identity 2morphism $1_{f}: f \rightarrow f$ for each 1-morphism $g$,

satisfying a few natural axioms. If we forget the 2-morphisms $\mathscr{C}$ should be an ordinary category. We adopt the convention that $i \in \mathscr{C}$ means that $i$ is an object in $\mathscr{C}$, and where $\mathscr{C}(\mathrm{i}, \mathrm{j})$ is the (full) 2-subcategory with

- objects: $\{i, j\}$,

- 1-morphisms: all 1-morphphisms $f, g: \mathrm{i} \rightarrow \mathrm{j}$ in $\mathscr{C}$,

- 2-morphisms: all 2-morphisms $\alpha, \beta: f \rightarrow g$ for $f, g$ 1-morphisms in $\mathscr{C}(\mathrm{i}, \mathrm{j})$.

Moreover, for each pair $\mathrm{i}$, j of objects we demand that $\mathscr{C}(\mathrm{i}, \mathrm{j})$ is a (small) category with objects the 1-morphisms $f, g: \mathrm{i} \rightarrow \mathrm{j}$ and morphisms all 2-morphisms between the appearing 1-morphisms. Before we state our last requirement we need to note that 2-morphisms can be composed in two ways. Besides the one we already implicitely mentioned, when $\alpha: f \rightarrow g$ and $\beta: g \rightarrow h$ composes to a 2-morphism $\alpha \beta: f \rightarrow h$, we also have a composition $\alpha_{1}: f_{1} \rightarrow g_{1}$ with $\alpha_{2}: f_{2} \rightarrow g_{2}$ which composes to a map $\left(\alpha_{1}, \alpha_{2}\right): f_{1} \circ f_{2} \rightarrow g_{1} \circ g_{2}$. We denote the first composition by $\circ_{1}$ and the latter by $\circ_{0}$. Now we require that $\circ_{0}$ is associative (whenever defined) and that

$$
\left(\alpha \circ_{0} \beta\right) \circ_{1}\left(\gamma \circ_{0} \delta\right)=\left(\alpha \circ_{1} \gamma\right) \circ_{0}\left(\beta \circ_{1} \delta\right)
$$

This axiom is frequently presented in the following diagrammatical form
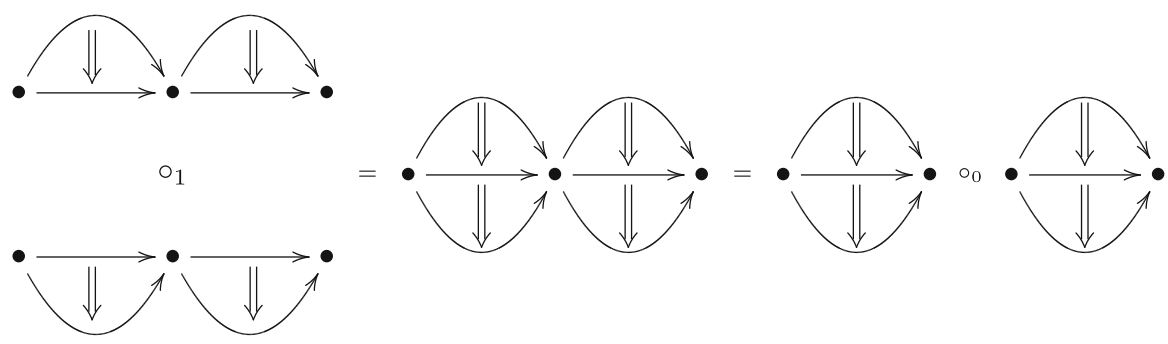

We refer, based on the diagram above, to the composition $\circ_{0}$ as horizontal, and the composition $\circ_{1}$ as vertical.

The canonical example of a 2-category is the category Cat of small categories where 
- objects are small categories,

- morphisms are categories where objects are functors and morphisms are natural transformations of functors,

- identities are the identity functors,

- composition is composition of functors.

Let $\mathbb{k}$ be a field. We will say that a 2 -category $\mathscr{C}$ is $\mathbb{k}$-admissible provided that,

- for any $i, j \in \mathscr{C}$, the category $\mathscr{C}(i, j)$ is $\mathbb{k}$-linear, idempotent split and KrullSchmidt,

- composition is $\mathbb{k}$-bilinear.

For example, let $A$ be a finite-dimensional associative algebra and $\mathcal{C}$ a small category equivalent to $A$-mod, then the 2 -full subcategory $\mathscr{R}_{(A, \mathcal{C})}$ of Cat with unique object $\mathcal{C}$ and whose 1-morphisms are right exact endofunctors on $\mathbb{C}$, is $\mathbb{k}$-admissible. The reason for this is the fact that $\mathscr{R}_{(A, \mathcal{C})}(\mathcal{C}, \mathcal{C})$ is equivalent to the category of $A$ - $A$-bimodules, see Bass (1968) for details.

\subsection{Grothendieck category of a $\mathbb{k}$-admissible 2-category}

Let $\mathcal{C}$ be an additive category. Then the split Grothendieck group $[\mathcal{C}]_{\oplus}$ of $\mathcal{C}$ is defined as the quotient of the free abelian group generated by $[X]$, where $X \in \mathcal{C}$, modulo the relations $[X]+[Y]-[Z]$ whenever $Z \cong X \oplus Y$. If $\mathcal{C}$ is idempotent split and KrullSchmidt, then $[\mathcal{C}]_{\oplus}$ is isomorphic to the free abelian group generated by isomorphism classes of indecomposable objects in $\mathcal{C}$.

Let $\mathscr{C}$ be a $\mathbb{k}$-admissible 2 -category. The associated Grothendieck category $[\mathscr{C}]_{\oplus}$, also called the decategorification of $\mathscr{C}$, is defined as the category such that

- $[\mathscr{C}]_{\oplus}$ has the same objects as $\mathscr{C}$,

- for $i, j \in[\mathscr{C}]_{\oplus}$, we have $[\mathscr{C}]_{\oplus}(i, j):=[\mathscr{C}(i, j)]_{\oplus}$,

- identity morphisms in $[\mathscr{C}]_{\oplus}$ are classes of the corresponding identity 1-morphisms in $\mathscr{C}$,

- composition in $[\mathscr{C}]_{\oplus}$ is induced from the composition in $\mathscr{C}$.

We note that the category $[\mathscr{C}]_{\oplus}$ is, by definition, preadditive, but not additive in general (as, in general, no coproduct of objects in $\mathscr{C}$ was assumed to exist).

Example 1 Let $S$ be a finite semigroup with an admissible partial order $\leq$. Define the 2-category $\mathscr{S}$ as follows:

- $\mathscr{S}_{S}$ has one object $i$;

- 1-morphisms in $\mathscr{S}_{S}$ are elements from $S$;

- composition of 1-morphisms is given by multiplication in $S$;

- for two 1-morphisms $s, t \in S$, we have

$$
\operatorname{Hom}_{\mathscr{S}}(s, t):=\left\{\begin{array}{l}
\varnothing, s \not \leq t ; \\
\left\{\mathbf{h}_{s, t}\right\}, s \leq t .
\end{array}\right.
$$


- vertical composition of 2-morphism is defined in the unique possible way which is justified by transitivity of $<$;

- horizontal composition of 2-morphism is defined in the unique possible way, which is justified by admissibility of $<$.

For a field $\mathbb{k}$, define the $\mathbb{k}$-linearization $\mathscr{S}_{\mathbb{k}}$ of $\mathscr{S}$ as follows, see [Grensing and Mazorchuk (2014b), Sect. 4.3] for details:

- $\mathscr{S}_{\mathbb{k}}$ has one object $i$;

- 1-morphisms in $\mathscr{S}_{\mathbb{k}}$ are formal finite direct sums of 1-morphisms in $\mathscr{S}$;

- 2-morphisms in $\mathscr{S}_{\mathbb{k}}$ are appropriate matrices whose entries are in $\mathbb{k} \mathbf{h}_{s, t}$;

- compositions in $\mathscr{S}_{\mathbb{K}}$ are induced from those in $\mathscr{S}$ using $\mathbb{k}$-bilinearity.

The 2-category $\mathscr{S}_{\mathbb{k}}$ is, by construction, $\mathbb{k}$-admissible. Moreover, the decategorification $\left[\mathscr{S}_{\mathbb{K}}\right]_{\oplus}$ of this 2-category

- has one object $i$;

- the endomorphism ring $\left[\mathscr{S}_{\mathbb{k}}\right]_{\oplus}(i, i)$ of the object $i$ is isomorphic to the integral semigroup ring $\mathbb{Z}[S]$.

\subsection{Finitary 2-categories}

A $\mathbb{k}$-admissible 2-category $\mathscr{C}$ is called finitary, see Mazorchuk and Miemietz (2011), provided that

- it has finitely many objects;

- it has finitely many indecomposable 1-morphisms, up to isomorphism;

- all $\mathbb{k}$-spaces of 2-morphisms are finite dimensional;

- all identity 1-morphisms are indecomposable.

For example, the category $\mathscr{S}_{\mathbb{K}}$ constructed in Example 1 is finitary (by construction and using the fact that $S$ is finite).

\subsection{Multisemigroups of $\mathbb{k}$-admissible 2-categories}

Let $\mathscr{C}$ be a $\mathbb{k}$-admissible 2-category. Consider the set $\mathcal{S}(\mathscr{C})$ of isomorphism classes of indecomposable 1-morphisms in $\mathscr{C}$. Recall, from Sect. 1, that setting, for two indecomposable 1-morphisms $\mathrm{F}$ and $\mathrm{G}$ in $\mathscr{C}$,

$$
[\mathrm{F}] *[\mathrm{G}]=\{[\mathrm{H}]: \mathrm{H} \text { is isomorphic to a direct summand of } \mathrm{F} \circ \mathrm{G}\} \text {, }
$$

defines on $\mathcal{S}(\mathscr{C})$ the structure of a multisemigroup. For example, for the category $\mathscr{S}_{\mathbb{K}}$ constructed in Example 1, the multisemigroup $\mathcal{S}(\mathscr{C})$ is canonically isomorphic to the semigroup $S$ (by sending [s] to $s$, for $s \in S$ ). In particular, in this case the multioperation defined by (1) is, in fact, single-valued and thus the prefix "multi" is redundant. 
Example 2 Consider the symmetric group $S_{3}$ as a Coxeter group with generators $s$ [standing for the elementary transposition $(1,2)]$ and $t$ [standing for the elementary transposition $(2,3)]$. Then

$$
S_{3}:=\{e, s, t, s t, t s, s t s\}
$$

where $s^{2}=t^{2}=e$ and $s t s=t s t$. Then we have the following Kazhdan-Lusztig basis in $\mathbb{Z}\left[S_{3}\right]$ :

$$
\begin{gathered}
\bar{e}:=e, \quad \bar{s}:=e+s, \quad \bar{t}:=e+t, \quad \overline{s t}:=e+s+t+s t, \\
\overline{t s}:=e+s+t+t s, \quad \overline{s t s}:=e+s+t+t s+s t+s t s .
\end{gathered}
$$

The multiplication table of the Kazhdan-Lusztig basis elements is given by:

\begin{tabular}{c||c|c|c|c|c|c}
$\cdot$ & $\bar{e}$ & $\bar{s}$ & $\bar{t}$ & $\overline{s t}$ & $\overline{t s}$ & $\overline{s t s}$ \\
\hline$\overline{\bar{e}}$ & $\bar{e}$ & $\bar{s}$ & $\bar{t}$ & $\overline{s t}$ & $\overline{t s}$ & $\overline{s t s}$ \\
\hline $\bar{s}$ & $\bar{s}$ & $2 \bar{s}$ & $\overline{s t}$ & $2 \overline{s t}$ & $\overline{s t s}+\bar{s}$ & $2 \overline{s t s}$ \\
\hline $\bar{t}$ & $\bar{t}$ & $\overline{t s}$ & $2 \bar{t}$ & $\overline{t s t}+\bar{t}$ & $2 \overline{t s}$ & $2 \overline{s t s}$ \\
\hline$\overline{s t}$ & $\overline{s t}$ & $\overline{s t s}+\bar{s}$ & $2 \overline{s t}$ & $2 \overline{t s t}+\overline{s t}$ & $2 \overline{s t s}+2 \bar{s}$ & $4 \overline{s t s}$ \\
\hline$\overline{t s}$ & $\overline{t s}$ & $2 \overline{t s}$ & $\overline{t s t}+\bar{t}$ & $2 \overline{t s t}+2 \bar{t}$ & $2 \overline{s t s}+\overline{t s}$ & $4 \overline{s t s}$ \\
$\overline{\overline{s t s}}$ & $\overline{s t s}$ & $2 \overline{s t s}$ & $2 \overline{s t s}$ & $4 \overline{s t s}$ & $4 \overline{s t s}$ & $6 \overline{s t s}$
\end{tabular}

Consider the 2-category $\mathscr{S}_{3}$ of Soergel bimodules over the coinvariant algebra of $S_{3}$ as detailed in, e.g., [Mazorchuk and Miemietz (2011), Sect. 7.1]. Consider the corresponding Grothendieck category $\left[\mathscr{S}_{3}\right]_{\oplus}$. Then the ring $\left[\mathscr{S}_{3}\right]_{\oplus}(i, i)$ is isomorphic to $\mathbb{Z}\left[S_{3}\right]$ where the isomorphism sends isomorphism classes of indecomposable 1morphisms in $\mathscr{S}_{3}$ to elements of the Kazhdan-Lusztig basis. This means that $\mathcal{S}\left[\mathscr{S}_{3}\right]$ can be identified with $S_{3}$ as a set. From (2) it follows that the multioperation $*$ on $\mathcal{S}\left[\mathscr{S}_{3}\right]$ is given by:

\begin{tabular}{c||c|c|c|c|c|c}
$\cdot$ & $e$ & $s$ & $t$ & $s t$ & $t s$ & $s t s$ \\
\hline \hline$e$ & $\{e\}$ & $\{s\}$ & $\{t\}$ & $\{s t\}$ & $\{t s\}$ & $\{s t s\}$ \\
\hline$s$ & $\{s\}$ & $\{s\}$ & $\{s t\}$ & $\{s t\}$ & $\{s t s, s\}$ & $\{s t s\}$ \\
\hline$t$ & $\{t\}$ & $\{t s\}$ & $\{t\}$ & $\{t s t, t\}$ & $\{t s\}$ & $\{s t s\}$ \\
\hline$s t$ & $\{s t\}$ & $\{s t s, s\}$ & $\{s t\}$ & $\{t s t, s t\}$ & $\{s t s, s\}$ & $\{s t s\}$ \\
\hline$t s$ & $\{t s\}$ & $\{t s\}$ & $\{t s t, t\}$ & $\{t s t, t\}$ & $\{$ sts,$t s\}$ & $\{s t s\}$ \\
\hline sts & $\{s t s\}$ & $\{s t s\}$ & $\{s t s\}$ & $\{s t s\}$ & $\{s t s\}$ & $\{s t s\}$
\end{tabular}

Here we see that the multioperation $*$ is genuinely multi-valued.

\subsection{Multisemigroups and decategorification}

Comparing (2) with (3), it is easy to see that the information encoded by the multisemigroup, that is (3), is not enough to recover the "associative algebra structure" 
which exists on the level of the Grothendieck decategorification presented in (2). The essential part of the lost information is the exact values of non-zero multiplicities with which indecomposable 1-morphisms appear in composition of two given indecomposable 1-morphisms.

One can say that the situation is even worse. Let us try to use (3) to define some associative algebra structure on the abelian group $\mathbb{Z}\left[S_{3}\right]$. The only reasonable guess would be to define, on generators, an operation $\diamond$ as follows:

$$
x \diamond y=\sum_{z \in x * y} z
$$

and then extend this to $\mathbb{Z}\left[S_{3}\right]$ by bilinearity. However, this is not associative, for example, $(s t s \diamond s t) \diamond s \neq s t s \diamond(s t \diamond s)$, indeed,

$$
(s t s \diamond s t) \diamond s=s t s \diamond s=s t s, \quad s t s \diamond(s t \diamond s)=s t s \diamond(s t s+s)=2 s t s .
$$

To have associativity, we should have considered $\mathbf{B}\left[S_{3}\right]$, where $\mathbf{B}$ is the Boolean semiring. This will be explained in detail later.

Therefore, if we want to define some discrete object which we could use to recover the associative algebra structure given by the Grothendieck decategorification, we need to keep track of multiplicities. This naturally leads to the notion of multisemigroups with multiplicities.

\section{Multisemigroups with multiplicities}

\subsection{Semirings}

A semiring is a weaker notion than that of a ring and the difference is that it is only required to form a commutative monoid (not a group) with respect to addition. More precisely, a unital semiring is a tuple $(R,+, \cdot, 0,1)$, where

- $R$ is a set;

- + and $\cdot$ are binary operations on $R$;

- 0 and 1 are two different elements of $R$.

These data is required to satisfy the following axioms.

- $(R,+, 0)$ is a commutative monoid with identity element 0 ;

- $(R, \cdot, 1)$ is a monoid with identity element 1 ;

- multiplication distributes over addition both from the left and from the right;

- $0 \cdot R=R \cdot 0=0$.

We refer to Golan (1999); Karner (1992) for more details.

Here are some examples of semirings:

- Any unital ring is a unital semiring.

- $\mathbb{Z}_{\geq 0}=(\{0,1,2,3, \ldots\},+, \cdot, 0,1)$. 
- The Boolean semiring $\mathbf{B}=(\{0,1\},+, \cdot, 0,1)$ with respect to the usual boolean addition and multiplication given by:

$$
\begin{array}{l||l|l}
+ & 0 & 1 \\
\hline \hline 0 & 0 & 1 \\
\hline 1 & 1 & 1
\end{array} \quad \text { and } \quad \begin{array}{l||l|l|l}
\quad & 0 & 1 \\
\hline 0 & 0 & 0 & 0 \\
\hline 1 & 0 & 1
\end{array}
$$

- The dual Boolean semiring $\mathbf{B}^{*}=(\{0,1\}, \cdot,+, 1,0)$ with respect to the boolean multiplication (as addition) and boolean addition (as multiplication).

- If $R$ is a semiring, then the set $\operatorname{Mat}_{n \times n}(R)$ of $n \times n$ matrices with coefficients in $R$ forms a semiring with respect to the usual addition and multiplication of matrices.

- For any nonempty set $X$, we have the semiring $\mathbf{B}_{X}:=\left(\mathbf{B}^{X}, \cup, \cap, \varnothing, X\right)$. This semiring is isomorphic to

$$
\prod_{x \in X} \mathbf{B}^{(x)}
$$

where $\mathbf{B}^{(x)}=\mathbf{B}$, a copy of the Boolean semiring $\mathbf{B}$ indexed by $x$.

Given two semirings $R$ and $R^{\prime}$, a homomorphism $\varphi: R \rightarrow R^{\prime}$ is a map from $R$ to $R^{\prime}$ such that

- $\varphi(r+s)=\varphi(r)+\varphi(s)$, for all $r, s \in R$;

- $\varphi(r \cdot s)=\varphi(r) \cdot \varphi(s)$, for all $r, s \in R$;

- $\varphi(0)=0$;

- $\varphi(1)=1$,

where we for simplicity suppress notation. Semirings and homomorphisms form a category, denoted by SRing.

\subsection{Complete semirings}

A commutative monoid $(S,+, 0)$ is called complete provided that it is equipped, for any indexing set $I$, with the sum operation $\sum_{i \in I}$ such that

- $\sum_{i \in \varnothing} r_{i}=0$;

- $\sum_{i \in\{j\}} r_{i}=r_{j}$

- $\sum_{j \in J} \sum_{i \in I_{j}} r_{i}=\sum_{s \in I} r_{s}$ when $\bigcup_{j \in J} I_{j}=I$ and $I_{j} \cap I_{j^{\prime}}=\varnothing$ for $j \neq j^{\prime}$.

We refer the reader to Hebisch (1992) for more details.

A semiring $(R,+, \cdot, 0,1)$ is called complete provided that

- $(R,+, 0)$ is a complete monoid; 
- multiplication distributes over all operations $\sum_{i \in I}$ on both sides, that is

$$
r \cdot\left(\sum_{i \in I} r_{i}\right)=\sum_{i \in I} r \cdot r_{i} \quad \text { and } \quad\left(\sum_{i \in I} r_{i}\right) \cdot r=\sum_{i \in I} r_{i} \cdot r
$$

Given two complete semirings $R$ and $R^{\prime}$, a homomorphism $\varphi: R \rightarrow R^{\prime}$ is a homomorphism of underlying semirings such that

$$
\varphi\left(\sum_{i \in I} r_{i}\right)=\sum_{i \in I}^{\prime} \varphi\left(r_{i}\right), \quad \text { for all } \quad r_{i} \in R .
$$

Complete semirings and homomorphisms form a subcategory in SRing, denoted by CSRing.

Here are some examples of complete semirings:

- Any complete lattice is a complete commutative semi-ring with respect to both choices $\{+, \cdot\}=\{\vee, \wedge\}$.

- $\left(\mathbf{B}^{X}, \cup, \cap, \varnothing, X\right)$, for some set $X$, where $\sum_{i \in I}$ is the usual union.

- The set of open sets for a topological space $X$, with respect to union and intersection.

- Unital quantales with join as addition and the underlying associative operation as multiplication.

- Integral tropical semiring $\left(\mathbb{Z}_{\geq 0} \cup\{-\infty\}\right.$, max, $\left.+,-\infty, 0\right)$, where $\sum_{i \in I}$ is just taking the supremum.

- The semiring $\left(\mathbb{Z}_{\geq 0} \cup\{\infty\},+\right.$, min, $\left.0, \infty\right)$, where the sum of infinitely many nonzero elements is set to be $\infty$.

- The semiring $\left(\mathbb{R}_{\geq 0} \cup\{\infty\},+, \cdot, 0,1\right)$, where $\sum_{i \in I}$ is defined as the supremum over all finite partial subsums.

- The semiring $\left(\mathbb{R}_{\geq 0} \cup\{\infty\},+, \cdot, 0,1\right)$, where any infinite sum of non-zero elements is defined to be $\infty$.

It is very tempting to add to the above the following "example": all cardinal numbers form a complete semiring with respect to the usual addition (disjoint union) and multiplication (Cartesian product) of cardinals. There is one problem with this "example", namely, the fact that cardinals do not form a set but, rather, a proper class. This problem can be overcome in an artificial and non-canonical way described in the next example. This example is separated from the rest due to its importance in what follows.

Example 3 For a fixed cardinal $\kappa$, let $\operatorname{Card}_{\kappa}$ denote the set of all cardinals which are not greater than $\kappa$. Then $\operatorname{Card}_{\kappa}$ has the structure of a complete semiring where

- addition (of any number of elements) is given by disjoint union with convention that all cardinals greater than $\kappa$ are identified with $\kappa$;

- multiplication is given by Cartesian product with convention that all cardinals greater than $\kappa$ are identified with $\kappa$.

Note that the Boolean semiring $\mathbf{B}$ is isomorphic to $\mathrm{Card}_{1}$. 


\subsection{Multisets}

Recall, see e.g. [Aigner (1979), p. 1], that a classical multiset is a pair $(A, \mu)$, where

- $A$ is a set;

- $\mu: A \rightarrow \mathbb{Z}_{\geq 0}$ is a function, called the multiplicity function.

A natural, more general, notion is that of a genuine multiset, which is a pair $(A, \mu)$, where

- $A$ is a set;

- $\mu$ is the multiplicity function from $A$ to the class of all cardinals.

\subsection{Multi-Booleans}

Recall that, given a base set $X$, the Boolean $\mathcal{B}(X)=\mathbf{B}^{X}$ of $X$ is the set of all subsets of $X$. This can be identified with the set of all functions from $X$ to the Boolean semiring B. In this way, $\mathcal{B}(X)$ gets the natural structure of a complete semiring with respect to the union and intersection of subsets. The additive identity is the empty subset while the multiplicative identity is $X$. Note that we can also consider the dual Boolean of $X$ which is the set of all functions from $X$ to the dual Boolean semiring $\mathbf{B}^{*}$. This gets the natural structure of a complete semiring with respect to the intersection and union of subsets. The additive identity is $X$ while the multiplicative identity is the empty subset.

The above point of view allows us to generalize the definition of the Boolean to multiset structures. Given a base set $X$, define the full multi-Boolean of $X$ to be the class of all functions from $X$ to the class of all cardinal numbers. To create any sensible theory, we need sets. This motivates the following definition.

Given a base set $X$ and a cardinal number $\kappa$, define the $\kappa$-multi-Boolean $\mathcal{B}_{\kappa}(X)$ of $X$ to be the set of all functions from $X$ to the complete semiring $\operatorname{Card}_{\kappa}$. By construction, $\mathcal{B}_{\kappa}(X)$ is equipped with the natural structure of a complete semiring. Also, we have $\mathcal{B}(X)=\mathcal{B}_{1}(X)$.

Clearly $\kappa=0$ would give us a singelton, on which no semi-ring structure exists. From now on we agree that any cardinal $\kappa$ in this paper is greater than or equal to 1 .

Recall that the union of multisets $X=\cup_{i} X_{i}$ contains an element $x$ as many times as the supremum of the number times $x$ appears in $X_{i}$. Now we have to make a choice. Either we permit $\infty$ as a coefficient, or we disallow some unions. We chose the former, as this only causes problems when considering multiset subtractions, which we are in any case not interested in. Similarly, the intersection of two multisets corresponds to infimum. Unfortunately, for $\kappa>1$, the natural complete semiring structure on $\mathcal{B}_{\kappa}(X)$ does not correspond to the usual set-theoretic notions of union and intersection of multisets. Note that these notions generalize union and intersection of normal sets. Indeed, the multiplicity analogue of the intersection of multisets is the arithmetic operation of taking minimum, while the multiplicity analogue of the union of multisets is the arithmetic operation of taking maximum. These differ from the usual addition and multiplication in $\operatorname{Card}_{\kappa}$, if $\kappa>1$. 


\subsection{Multisemigroups with multiplicity}

Now we are ready to present our main definition. Let $\kappa$ be a fixed cardinal. A multisemigroup with multiplicities bounded by $\kappa$ is a pair $(S, \mu)$, where

- $S$ is a non-empty set;

- $\mu: S \times S \rightarrow \mathcal{B}_{\kappa}(S)$, written $(s, t) \mapsto \mu_{s, t}: S \rightarrow \operatorname{Card}_{\kappa}$;

such that the following distributivity requirement is satisfied: for all $r, s, t \in S$, we have

$$
\sum_{i \in S} \mu_{s, t}(i) \mu_{r, i}=\sum_{j \in S} \mu_{r, s}(j) \mu_{j, t}
$$

We note that here, for a cardinal $\lambda$ and a function $\nu: S \rightarrow \operatorname{Card}_{\kappa}$, by $\lambda \nu$ we mean the function from $S$ to $\operatorname{Card}_{\kappa}$ defined as

$$
\lambda v=\sum_{i \in \lambda} v,
$$

or, in other words, this is just adding up $\lambda$ copies of $\nu$.

The informal explanation for the requirement (4) is as follows: the left hand side corresponds to the "product" $r *(s * t)$. Here $s * t$ gives $\mu_{s, t}$, which counts every $i \in S$ with multiplicity $\mu_{s, t}(i)$. The result of $r *(s * t)$, written when we distribute $r *$ over all such $i \in S$ and taking multiplicities into account, gives exactly the left hand side in (4). Similarly, the right hand side corresponds to the "product" $(r * s) * t$.

If $\kappa$ is clear from the context, we will sometimes use the shorthand multi-multisemigroup instead of "multisemigroup with multiplicities bounded by $\kappa$ ".

Here are some easy examples of multisemigroups with multiplicities:

- A usual multisemigroup is a multisemigroup with multiplicities bounded by one.

- For any $\kappa$ and any $\lambda<\kappa$, the set $\{a\}$ has the structure of a multisemigroup with multiplicities bounded by $\kappa$, if we set $\mu_{a, a}=\lambda$. Moreover, these exhaust all such structures on $\{a\}$.

Here is a more involved example:

Example 4 Let $A$ be a finite dimensional $\mathbb{R}$-algebra with a fixed basis $\left\{a_{i}: i \in I\right\}$ such that $a_{i} \cdot a_{j}=\sum_{s \in I} \mu_{i, j}^{s} a_{s}$ and all $\mu_{i, j}^{s} \in \mathbb{Z}_{\geq 0}$. Then $(I, \mu)$, where we define $\mu_{i, j}(s):=\mu_{i, j}^{s}$, is a multisemigroup with multiplicities bounded by $\omega$, the first infinite cardinal. This follows from the associativity of multiplication in $A$ via the computation

$$
\sum_{s} \sum_{t} \mu_{i, j}^{t} \mu_{t, k}^{s} a_{s}=\left(a_{i} \cdot a_{j}\right) \cdot a_{k}=a_{i} \cdot\left(a_{j} \cdot a_{k}\right)=\sum_{x} \sum_{y} \mu_{i, y}^{x} \mu_{j, k}^{y} a_{x},
$$

which is equivalent to (4) in this case since basis elements are linearly independent.

Let $(S, \mu)$ and $\left(S^{\prime}, \mu^{\prime}\right)$ be two multisemigroups with multiplicities bounded by $\kappa$. We will say that they are isomorphic provided that there is a bijection $\varphi: S \rightarrow S^{\prime}$ such that $\mu^{\prime}=\varphi \circ \mu$. 
Let $(S, \mu)$ be a multisemigroups with multiplicities bounded by $\kappa$. Let $S^{\diamond}$ denote the set of all words in the alphabet $S$ of length at least two. Define the map

$$
\bar{\mu}: S^{\diamond} \rightarrow \mathcal{B}_{\kappa}(S)
$$

recursively as follows:

1. $\bar{\mu}_{s t}=\mu_{s, t}$, if $s, t \in S$;

2. if $w=s x$, where $x$ has length at least two, then set

$$
\bar{\mu}_{w}(t):=\sum_{a \in S} \bar{\mu}_{x}(a) \mu_{s, a}(t)
$$

The definition of $\bar{\mu}$ does not really depend on our choice of prefix above (in contrast to suffix), as is clear from the following statement.

Proposition 5 If $w \in S^{\diamond}$ has the form $w=x s$, where $x$ has length at least $t w o$, then

$$
\bar{\mu}_{w}(t):=\sum_{a \in S} \bar{\mu}_{x}(a) \mu_{a, s}(t)
$$

Proof Let $w=s_{1} s_{2} \ldots s_{k}$, where $k \geq 3$. Then the recursive procedure in (5) results in

$$
\sum_{i_{1} \in S} \sum_{i_{2} \in S} \cdots \sum_{i_{k-2} \in S} \mu_{s_{1}, i_{1}}(t) \mu_{s_{2}, i_{2}}\left(i_{1}\right) \cdots \mu_{s_{k-2}, i_{k-2}}\left(i_{k-3}\right) \mu_{s_{k-1}, s_{k}}\left(i_{k-2}\right) .
$$

The recursive procedure in (6) results in

$$
\sum_{j_{1} \in S} \sum_{j_{2} \in S} \cdots \sum_{j_{k-2} \in S} \mu_{s_{1}, s_{2}}\left(j_{1}\right) \mu_{j_{1}, s_{3}}\left(j_{2}\right) \cdots \mu_{j_{k-3}, s_{k-1}}\left(j_{k-2}\right) \mu_{j_{k-2}, s_{k}}(t) .
$$

The expression (7) it transferred to (8) using a repetitive application of (4). The claim follows.

\subsection{Finitary multisemigroups with multiplicities}

We will say that a multisemigroup $(S, \mu)$ with multiplicities bounded by $\kappa$ is finitary provided that

- $\kappa=\aleph_{0}$;

- $\mu_{r, s}(t) \neq \aleph_{0}$ for all $r, s, t \in S$;

- $\left|\left\{t \in S: \mu_{r, s}(t) \neq 0\right\}\right|<\aleph_{0}$ for all $r, s \in S$.

\subsection{Multi-multisemigroup of a $\mathbb{k}$-admissible 2-category}

Let $\mathscr{C}$ be a $\mathbb{k}$-admissible 2-category. Consider the set $\mathcal{S}(\mathscr{C})$ of isomorphism classes of indecomposable 1-morphisms in $\mathscr{C}$. For $F, G, H \in \mathcal{S}(\mathscr{C})$, define $\mu_{F, G}(H)$ to be the multiplicity of $H$ as a direct summand in the composition $F \circ G$. 
Theorem 6 The construct $(\mathcal{S}(\mathscr{C}), \mu)$ is a finitary multisemigroup with multiplicities.

Proof We only have to check (4) in this case, as the rest follows by construction from $\mathbb{k}$-admissibility of $\mathscr{C}$. For $F, G, H, K \in \mathcal{S}(\mathscr{C})$, the multiplicity of $K$ in $(F \circ G) \circ H$ is given by

$$
\sum_{Q \in \mathcal{S}(\mathscr{C})} \mu_{F, G}(Q) \mu_{Q, H}(K)
$$

In turn, the multiplicity of $K$ in $F \circ(G \circ H)$ is given by

$$
\sum_{P \in \mathcal{S}(\mathscr{C})} \mu_{F, P}(K) \mu_{G, H}(P)
$$

As $(F \circ G) \circ H \cong F \circ(G \circ H)$ and $\mathcal{S}(\mathscr{C})$ is Krull-Schmidt, we have

$$
\sum_{Q \in \mathcal{S}(\mathscr{C})} \mu_{F, G}(Q) \mu_{Q, H}(K)=\sum_{P \in \mathcal{S}(\mathscr{C})} \mu_{F, P}(K) \mu_{G, H}(P),
$$

which proves (4) in this case.

Example 7 For the 2-category $\mathscr{S}_{3}$ in Example 2, the multi-multisemigroup structure on $\mathcal{S}(\mathscr{C})$ is fully determined by (2). For instance, the function $\mu_{\overline{s t}, \overline{s t}}$ has the following values:

$$
\begin{array}{c||c|c|c|c|c|c}
x: & \bar{e} & \bar{s} & \bar{t} & \overline{s t} & \overline{t s} & \overline{s t s} \\
\hline \hline \mu_{\overline{s t}, \overline{s t}}(x): & 0 & 0 & 0 & 1 & 0 & 2 .
\end{array}
$$

The function $\mu_{\overline{t s}}, \overline{s t s}$ has the following values:

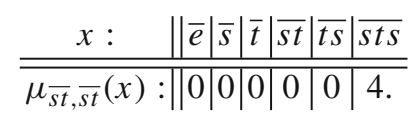

\section{Multi-multisemigroup vs multisemigroup and decategorification}

\subsection{Multi-multisemigroup vs multisemigroup}

Consider the canonical surjective semiring homomorphism $\Phi: \operatorname{Card}_{\omega} \rightarrow \operatorname{Card}_{1} \cong \mathbf{B}$ defined by

$$
\Phi(x)= \begin{cases}0, & x=0 \\ 1, & \text { otherwise }\end{cases}
$$

As usual, we identify subsets in a set $X$ with $\mathbf{B}^{X}$. The following proposition says that the multi-multisemigroup of $\mathscr{C}$ has enough information to recover the multisemigroup of $\mathscr{C}$. 
Proposition 8 Let $\mathscr{C}$ be a $\mathbb{k}$-admissible 2-category. Then, for any $[F],[G] \in \mathcal{S}(\mathscr{C})$, we have

$$
[F] *[G]=\Phi \circ \mu_{F, G} .
$$

Proof The left hand side is, by definition, the set of isomorphism classes of indecomposable 1-morphisms $H$ such that $H$ appears (up to isomorphism) in $F \circ G$. Before we apply $\Phi$, the right hand side precisely records all indecomposable 1-morphisms appearing in $F \circ G$ with multiplicities. $\Phi$ forgets multiplicities, but preserves the rest of the structure. Therefore the left and right hand sides coincide for all $F$ and $G$.

\subsection{The algebra of a finitary multi-multisemigroup}

Let $(S, \mu)$ be a finitary multi-multisemigroup. For a fixed commutative unital ring $\mathbb{k}$, consider the free $\mathbb{k}$-module $\mathbb{k}[S]$ with basis $S$. Define on $\mathbb{k}[S]$ a $\mathbb{k}$-bilinear binary multiplication - by setting, for $s, t \in S$,

$$
s \cdot t:=\sum_{r \in S} \mu_{s, t}(r) r
$$

Proposition 9 The construct $(\mathbb{k}[S], \cdot)$ is an associative $\mathbb{k}$-algebra.

Proof We need to show that $(r \cdot s) \cdot t=r \cdot(s \cdot t)$, for all $r, s, t \in S$. Using (9) and $\mathbb{k}$-bilinearity of $\cdot$, this reduces exactly to the axiom (4).

\subsection{Grothendieck ring of a $\mathbb{k}$-admissible 2-category}

Let $\mathscr{C}$ be a small $\mathbb{k}$-admissible 2-category. The Grothendieck ring $\operatorname{Gr}(\mathscr{C})$ of $\mathscr{C}$ is defined as follows:

- elements of $\operatorname{Gr}(\mathscr{C})$ are elements in the free abelian group generated by isomorphism classes of indecomposable 1-morphisms;

- addition in $\operatorname{Gr}(\mathscr{C})$ is the obvious addition inside the free abelian group;

- multiplication in $\operatorname{Gr}(\mathscr{C})$ is induced from composition in $\mathscr{C}$ using biadditivity.

The ring $\operatorname{Gr}(\mathscr{C})$ is unital if and only if $\mathscr{C}$ has finitely many objects. Otherwise it is locally unital, where local units correspond to (summands of) the identity 1-morphisms in $\mathscr{C}$.

An alternative way to look at $\operatorname{Gr}(\mathscr{C})$ is to understand it as the ring associated with the preadditive category $[\mathscr{C}]_{\oplus}$ in the obvious way. Conversely, $[\mathscr{C}]_{\oplus}$ is the variation of the ring $\operatorname{Gr}(\mathscr{C})$ which has several objects, cf. Mitchell (1972).

\subsection{Multi-multisemigroup vs decategorification}

Our main observation in this subsection is the following connection between the multimultisemigroup of a finitary 2-category and the Grothendieck ring of this category. 
Proposition 10 Let $\mathscr{C}$ be a finitary 2-category and $\mathbb{k}$ a field. Then there is a canonical isomorphism of $\mathbb{k}$-algebras,

$$
\mathbb{k} \otimes_{\mathbb{Z}} \operatorname{Gr}(\mathscr{C}) \cong \mathbb{k}[\mathcal{S}(\mathscr{C})]
$$

Proof We define the map $\psi: \mathbb{k} \otimes_{\mathbb{Z}} \operatorname{Gr}(\mathscr{C}) \rightarrow \mathbb{k}[\mathcal{S}(\mathscr{C})]$ as the $\mathbb{k}$-linear extension of the map which sends an isomorphism class of indecomposable 1-morphisms in $\mathscr{C}$ to itself. This map is, clearly, bijective. Moreover, it is a homomorphism of rings since, on both sides, the structure constants with respect to the $\mathbb{k}$-basis, consisting of isomorphism class of indecomposable 1-morphisms in $\mathscr{C}$, are given by non-negative integers $\mu_{F, G}(H)$ as defined in Sect. 3.5. The claim of the proposition follows.

Altogether, for a finitary 2-category $\mathscr{C}$, we have the following picture

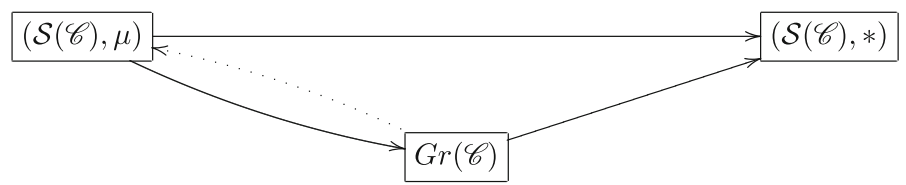

where arrow show in which direction we can recover information.

\section{Some explicit examples of multi-multisemigroups of finitary 2-categories}

\subsection{Projective functors for finite dimensional algebras}

Let $\mathbb{k}$ be an algebraically closed field and $A$ a connected, basic, non-semi-simple, finite dimensional, unital $\mathbb{k}$-algebra. Let $\mathcal{C}$ be a small category equivalent to $A$-mod. Following [Mazorchuk and Miemietz (2011), Sect. 7.3], we define the 2-category $\mathscr{C}_{A}$ as a subcategory in Cat (not full) such that:

- $\mathscr{C}_{A}$ has one object $i$, which we identify with $\mathcal{C}$;

- 1-morphisms in $\mathscr{C}_{A}$ are functors isomorphic to direct sums of the identity functors and functors of tensoring with projective $A$-bimodules;

- 2-morphisms in $\mathscr{C}_{A}$ are natural transformations of functors.

Note that all 1-morphisms in $\mathscr{C}_{A}$ are, up to isomorphism, functors of tensoring with $A$-bimodules. For simplicity we will just use certain bimodules to denote the corresponding isomorphism classes of 1-morphisms.

Let $1=e_{1}+e_{2}+\cdots+e_{k}$ be a decomposition of $1 \in A$ into a sum of primitive, pairwise orthogonal idempotents. Then indecomposable 1-morphisms in $\mathscr{C}_{A}$ correspond to bimodule

$$
\nVdash:=A, \quad F_{i, j}:=A e_{i} \otimes_{\mathbb{R}} e_{j} A, \text { where } i, j=1,2, \ldots, k .
$$

The essential part of the composition in $\mathscr{C}_{A}$ is given by

$$
F_{i, j} \circ F_{i^{\prime}, j^{\prime}}=F_{i, j^{\prime}}^{\oplus \operatorname{dim} e_{j} A e_{i^{\prime}}},
$$


as follows from the computation

$$
A e_{i} \otimes_{\mathbb{k}} e_{j} A \otimes_{A} A e_{i^{\prime}} \otimes_{\mathbb{k}} e_{j^{\prime}} A \cong A e_{i} \otimes_{\mathbb{k}} e_{j^{\prime}} A^{\oplus \operatorname{dim} e_{j} A e_{i^{\prime}}}
$$

The above implies that

$$
\mathcal{S}\left(\mathscr{C}_{A}\right)=\left\{\mathbb{1}, F_{i, j}: i, j=1,2, \ldots, k\right\}
$$

and the multiplicity function defining the multi-multisemigroup structure on $\mathcal{S}\left(\mathscr{C}_{A}\right)$ is given by

$$
\mu_{F, G}(H)= \begin{cases}1, & H=G \text { and } F=\mathbb{1} ; \\ 1, & H=F \text { and } G=\mathbb{1} ; \\ \operatorname{dim}_{j} A e_{i^{\prime}}, & F=F_{i, j}, G=F_{i^{\prime}, j^{\prime}}, H=F_{i, j^{\prime}} ; \\ 0, & \text { otherwise. }\end{cases}
$$

Note also that, in this case, the multioperation in the multisemigroup $\left(\mathcal{S}\left(\mathscr{C}_{A}\right), *\right)$ is, at most, single valued. By adding, if necessary, an external element 0 , we can turn $\left(\mathcal{S}\left(\mathscr{C}_{A}\right), *\right)$ into a genuine semigroup.

\subsection{Soergel bimodules for finite Coxeter groups}

Another prominent example of a finitary 2-category is the finitary 2-category of Soergel bimodules. Let $W$ be a finite Coxeter group with a fixed geometric representation. To these data, one associates the so-called 2-category $\mathscr{S}_{W}$ of Soergel bimodules over the coinvariant algebra of the geometric representation, see Soergel (2007) and [Mazorchuk and Miemietz (2011), Sect. 7.1]. This is a finitary 2-category. This 2-category categorifies the integral group ring of $W$ in the sense that there is an isomorphism between the ring $[\mathscr{C}]_{\oplus}(i, i)$ and the ring $\mathbb{Z}[W]$ given in terms of the Kazhdan-Lusztig basis in $\mathbb{Z}[W]$, see Kazhdan and Lusztig (1979). Therefore the multi-multisemigroup $\left(\mathcal{S}\left(\mathscr{S}_{W}\right), \mu\right)$ records exactly the information about the structure constants of the ring $\mathbb{Z}[W]$ with respect to the Kazhdan-Lusztig basis. As far as we know, there is no explicit combinatorial formula for such structure constants, however, they can be determined using a recursive algorithm.

In the special case of a Dihedral group $D_{n}$, where $n \geq 3$,

$$
W=D_{n}=\left\{s, t: s^{2}=t^{2}=(s t)^{n}=e\right\},
$$

the Kazhdan-Lusztig basis has particularly simple form. Elements of the group $D_{n}$ can be listed as

$$
D_{n}=\left\{e, s, t, s t, t s, \ldots, w_{0}\right\}
$$

where $w_{0}=s t s t \ldots=t s t s \ldots$, where the length of both words is $n$. Then, for each $w \in D_{n}$, the corresponding Kazhdan-Lusztig basis element $\underline{w} \in \mathbb{Z}\left[D_{n}\right]$ is the sum of $w$ with all elements of strictly smaller length. 
Let $\mathbf{l}: D_{n} \rightarrow \mathbb{Z}_{\geq 0}$ be the length function with respect to generators $s$ and $t$. A direct calculation then shows that

$$
\underline{s} \cdot \underline{w}=\left\{\begin{array}{ll}
2 \underline{w}, & \mathbf{l}(s w)<\mathbf{l}(w) ; \\
\underline{s w}, & w=e \text { or } w=t ; \\
\underline{s w}+\underline{t w}, & \text { otherwise; }
\end{array} \quad \underline{t} \cdot \underline{w}= \begin{cases}2 \underline{w}, & \mathbf{l}(t w)<\mathbf{l}(w) ; \\
\underline{t w}, & w=e \text { or } w=s \\
\underline{s w}+\underline{t w}, & \text { otherwise. }\end{cases}\right.
$$

This already shows that the multi-multisemigroup structure is non-trivial in the sense that it is not reducible to a multisemigroup structure. The above formulae determine the multiplicity functions $\mu_{s, w}$ and $\mu_{t, w}$. As any element in $D_{n}$ is a product of $s$ and $t$, all remaining multiplicity functions can be determined inductively. However, we do not know of any explicit formulae. For $n=3$, the answer is given in (2). More information on the $D_{n}$ case can be found in Elias (2011).

\subsection{Catalan monoid}

Let $n$ be a positive integer. Consider the path algebra $A=A_{n}$ over $\mathbb{C}$ of the quiver

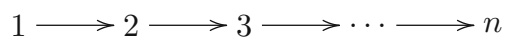

Let $\mathcal{C}$ be a small category equivalent to $A$-mod. Following Grensing and Mazorchuk (2014a), we define the 2-category $\mathscr{G}_{n}$ as a subcategory in Cat (not full) such that:

- $\mathscr{C}_{A}$ has one object $i$, which we identify with $\mathcal{C}$;

- 1-morphisms in $\mathscr{C}_{A}$ are functors isomorphic to direct sums of functors of tensoring with subbimodules of ${ }_{A} A_{A}$;

- 2-morphisms in $\mathscr{C}_{A}$ are natural transformations of functors.

The main result of Grensing and Mazorchuk (2014a) asserts that the multisemigroup $\mathcal{S}\left(\mathscr{G}_{n}\right)$ (with added zero) is isomorphic to the Catalan monoid $\mathrm{C}_{n+1}$ of all orderpreserving and order-decreasing transformation of a finite chain with $n+1$ elements. In particular, the multisemigroup $\mathcal{S}\left(\mathscr{G}_{n}\right)$ is a semigroup.

Moreover, in Grensing and Mazorchuk (2014a) it is also shown that the composition of indecomposable 1-morphism in $\mathscr{G}_{n}$ is indecomposable (or zero). This means that, in this case, the multi-multisemigroup structure on $\mathcal{S}\left(\mathscr{G}_{n}\right)$ coincides with the multisemigroup structure.

A similar phenomenon was observed in some other cases in Zhang (2015a,b).

\section{Multi-multisemigroups with different multiplicities}

\subsection{Cardinal reduction}

Let $\lambda<\kappa$ be two cardinal numbers. Then there is a canonical homomorphism

$$
\Phi_{\lambda, \kappa}: \operatorname{Card}_{\kappa} \rightarrow \operatorname{Card}_{\lambda}
$$


of complete semirings defined as follows:

$$
\Phi_{\lambda, \kappa}(v)= \begin{cases}v, & v \leq \lambda \\ \lambda, & \text { otherwise }\end{cases}
$$

Proposition 11 Let $(S, \mu)$ be a multisemigroup with multiplicities bounded by $\kappa$. Then $\left(S, \Phi_{\lambda, \kappa} \circ \mu\right)$ is a multisemigroup with multiplicities bounded by $\lambda$.

Proof The axiom (4) in the new situation (for $\lambda$ ) follows from the axiom (4) in the old situation (for $\kappa$ ) by applying the homomorphism $\Phi_{\lambda, \kappa}$ of complete semirings to both sides.

A special case of this construction was mentioned in Sect. 4.1, in that case $\kappa=\omega$ and $\lambda=1$. A natural question is whether this construction is "surjective" in the sense that any multisemigroups with multiplicities bounded by $\lambda$ can be obtained in this way from a multisemigroups with multiplicities bounded by $\kappa$. If $\lambda=1$, the answer is yes due to the following construction:

Let $\kappa$ be a nonzero cardinal numbers. Then there is a canonical homomorphism

$$
\Psi_{\kappa}: \operatorname{Card}_{1} \cong \mathbf{B} \rightarrow \operatorname{Card}_{\kappa}
$$

which sends 0 to 0 and also sends 1 to $\kappa$. Given a multisemigroup $(S, *)$, we thus may define

$$
\mu_{s, t}(r):= \begin{cases}0, & r \notin s * t \\ \kappa, & r \in s * t\end{cases}
$$

In other words, we define $\mu$ as the composition of $*$ followed by $\Psi_{\kappa}$. Similarly to the proof of Proposition 11 we thus get that $(S, \mu)$ is a multisemigroups with multiplicities bounded by $\kappa$. As the homomorphism $\Phi_{1, \kappa} \circ \Psi_{\kappa}$ is the identity on $\mathbf{B}$, we obtain $(S, *)=\left(S, \Phi_{1, \kappa} \circ \mu\right)$.

\subsection{Finitary cardinal reduction}

To avoid degenerate examples above, it is natural to rephrase the question as follows: Given a multisemigroup $(S, *)$, whether there is a finitary multi-multisemigroup $(S, \mu)$ such that $(S, *)=\left(S, \Phi_{1, \omega} \circ \mu\right)$. The following example shows that this is, in general, not the case.

Proposition 12 (i) There is a multisemigroup $(\{a, b\}, *)$ with the following multiplication table:

$$
\begin{array}{c||c|c}
* & a & b \\
\hline \hline a & \{a\} & \{a, b\} \\
\hline b & \{a, b\} & \{a, b\}
\end{array}
$$


(ii) The multisemigroup $(\{a, b\}, *)$ is not of the form $\left(S, \Phi_{1, \omega} \circ \mu\right)$, for any finitary multisemigroup $(S, \mu)$ with multiplicities.

Proof It is clear that the multiplication table (10) defines a multisemigroup, as any product $x * y * z$ is $a$ if $x=y=z=a$, and $\{a, b\}$ otherwise.

Now assume that $(\{a, b\}, \mu)$ is a finitary multisemigroup with multiplicities. Then $\mu_{a, b}(a) \neq 0$ because $a \in a * b$, moreover, we have $\mu_{a, b}(b) \neq 0$ as $b \in a * b$.

We want to compute $\bar{\mu}_{a a b}(a)$ in two different ways, namely, using the decompositions $(a a) b$ and $a(a b)$. In the first case, we get $\bar{\mu}_{a a b}(a)=\mu_{a, a}(a) \mu_{a, b}(a)$. In the second case, we obtain $\bar{\mu}_{a a b}(a)=\mu_{a, a}(a) \mu_{a, b}(a)+\mu_{a, b}(b) \mu_{a, b}(a)$. As both $\mu_{a, b}(a) \neq 0$ and $\mu_{a, b}(b) \neq 0$, we get a contradiction. The claim follows.

\subsection{Deformation of multisemigroups}

Let $(S, *)$ be a finite multisemigroup. A finitary multi-multisemigroup $(S, \mu)$ such that $(S, *)=\left(S, \Phi_{1, \omega} \circ \mu\right)$ is called a deformation of $(S, *)$. As we saw above, not every finite multisemigroup admits a deformation. It would be interesting to find some sufficient and necessary conditions for a multisemigroup to admit a non-trivial deformation. Ideally, it would be really interesting to find some way to describe all possible deformations of a given multisemigroup. The following is a corollary from the result in the previous section.

Corollary 13 Let $(S, *)$ be a multisemigroup containing two different elements a and $b$ such that $a * a=\{a\}$ and $\{a, b\} \subset a * b$ or $\{a, b\} \subset b * a$. Then $(S, *)$ does not admit any deformation.

Proof In the case $\{a, b\} \subset a * b$, the claim follows from the arguments in the proof of Proposition 12. In case $\{a, b\} \subset b * a$ the proof is similar.

Another obvious observation is the following.

Proposition 14 Let $\mathscr{C}$ be a finitary 2-category. Then $(\mathcal{S}(\mathscr{C}), *)$ admits a deformation.

Proof By construction, $(\mathcal{S}(\mathscr{C}), \mu)$ is a deformation of $(\mathcal{S}(\mathscr{C}), *)$.

\subsection{Connection to twisted semigroup algebras}

In case a finite multisemigroup $(S, *)$ is a semigroup, deformations of $(S, *)$ can be understood as integral twisted semigroup algebras in the sense of Guo and Xi (2009). Indeed, according to the above definition, a deformation of a finite semigroup $(S, *)$ is given by a map

$$
\mu: S \times S \rightarrow \mathbb{Z}_{\geq 0}
$$

which satisfies axiom (4) (the associativity axiom). This is a special case of the definition of twisted semigroup algebras, see [Guo and Xi (2009), Sect. 3] or [Wilcox (2007), Eq. (1)]. Typical examples of semigroups which admit non-trivial twisted semigroup algebras (and hence also non-trivial deformations) are various diagram algebras, see Martin and Mazorchuk (2013); Wilcox (2007) for details. 


\section{Multi-multisemigroups and modules over complete semirings}

\subsection{Modules over semirings}

Let $R$ be a unital semiring. A (left) $R$-module is a commutative monoid $(M,+, 0)$ together with the map $: R \times M \rightarrow M$, written $(r, m) \mapsto r \cdot m$, such that

- $(r s) \cdot m=r \cdot(s \cdot m)$, for all $r, s \in R$ and $m \in M$;

- $(r+s) \cdot m=r \cdot m+s \cdot m$, for all $r, s \in R$ and $m \in M$;

- $r \cdot(m+n)=r \cdot m+r \cdot n$, for all $r \in R$ and $m, n \in M$;

- $0 \cdot m=0$, for all $m \in M$;

- $1 \cdot m=m$, for all $m \in M$.

We refer, for example, to Johnson and Manes (1970) for more details. For instance, the multiplication on $R$ defines on $R$ the structure of a left $R$-module ${ }_{R} R$, called the regular module.

\subsection{Modules over complete semirings}

Let $R$ be a complete unital semiring. A (left) complete $R$-module is an $R$-module $(M,+, 0, \cdot)$ such that

- $M$ is complete;

- $r \cdot \sum_{i \in I} m_{i}=\sum_{i \in I} r \cdot m_{i}$, for all $r \in R$ and $m_{i} \in M$;

- $\left(\sum_{i \in I} r_{i}\right) \cdot m=\sum_{i \in I} r_{i} \cdot m$, for all $r_{i} \in R$ and $m \in M$.

For example, the regular $R$-module is complete. Another important example of a complete $R$-module is the following.

Example 15 Let $R$ be a complete unital semiring and $X$ a non-empty set. Then the set $R^{X}$ of all functions $f: X \rightarrow R$ from $X$ to $R$ is a complete abelian group with respect to component-wise addition

$$
\left(\sum_{i \in I} f_{i}\right)(x):=\sum_{i \in I} f_{i}(x)
$$

moreover, it has the natural structure of a complete $R$-module given by componentwise multiplication with elements in $R$,

$$
(r \cdot f)(x)=r \cdot f(x) .
$$

This module has, as an incomplete submodule, the set of all functions in $R^{X}$ with finitely many non-zero values. 


\subsection{Algebras over complete semirings}

For a complete unital semiring $R$ and a non-empty set $X$, consider the complete $R$-module $R^{X}$ as in Example 15 above. An algebra structure on $R^{X}$ is a map • : $R^{X} \times R^{X} \rightarrow R^{X}$ such that, for all $f_{i}, f, g, h \in R^{X}$, we have

$$
\begin{gathered}
\left(\sum_{i \in I} f_{i}\right) \bullet g=\sum_{i \in I} f_{i} \bullet g ; \\
g \bullet\left(\sum_{i \in I} f_{i}\right)=\sum_{i \in I} g \bullet f_{i} ; \\
f \bullet(g \bullet h)=(f \bullet g) \bullet h .
\end{gathered}
$$

For example, if $X=\{a\}$, then $R^{X}=R$ and the multiplication - on $R$ defines on $R$ the structure of a complete $R$-algebra.

\subsection{Connection to multi-multisemigroups}

If $R$ is a semiring and $X$ a set, then, for $x \in X$, we denote by $\chi_{x}: X \rightarrow R$ the indicator function of $x$ defined as follows:

$$
\chi_{x}(y)= \begin{cases}1, & x=y \\ 0, & x \neq y\end{cases}
$$

Our main result in this section is the following:

Theorem 16 (i) Let $\kappa$ be a cardinal and $(S, \mu)$ be a multisemigroup with multiplicities bounded by $\kappa$. Then Card $_{\kappa}^{S}$ has a unique structure of a complete Card ${ }_{\kappa}$-algebra such that $\chi_{s} \bullet \chi_{t}=\mu_{s, t}$, for all $s, t \in S$.

(ii) Conversely, any complete $\operatorname{Card}_{\kappa}^{S}$-algebra $\left(\operatorname{Card}_{\kappa}^{S}, \bullet\right)$ defines a unique structure of a multisemigroup with multiplicities bounded by $\kappa$ on $S$ via $\mu_{s, t}:=\chi_{s} \bullet \chi_{t}$, for $s, t \in S$.

Proof To prove claim (i), we first note that uniqueness would follow directly from existence as any element in $\operatorname{Card}_{\kappa}^{S}$ can be written as a sum, over $S$, of indicator functions. To prove existence, we note that each function can be uniquely written as a sum, over $S$, of indicator functions. Therefore, there is a unique way to extend $\chi_{s} \bullet \chi_{t}:=\mu_{s, t}$, for $s, t \in S$, to a map $\bullet: \operatorname{Card}_{\kappa}^{S} \times \operatorname{Card}_{\kappa}^{S} \rightarrow \operatorname{Card}_{\kappa}^{S}$ which satisfies (11) and (12). Using (11) and (12), it is enough to check the axiom (13) for the indicator functions, where it is equivalent to the axiom (4), by definition. This proves claim (i).

To prove claim (ii), we only need to check the axiom (4). This axiom follows from the axiom (13) applied to the indicator functions. This completes the proof.

Theorem 16 suggests that one could define multisemigroups with multiplicities from any complete semiring, not necessarily $\operatorname{Card}_{\kappa}$. 
Open Access This article is distributed under the terms of the Creative Commons Attribution 4.0 International License (http://creativecommons.org/licenses/by/4.0/), which permits unrestricted use, distribution, and reproduction in any medium, provided you give appropriate credit to the original author(s) and the source, provide a link to the Creative Commons license, and indicate if changes were made.

\section{References}

Aigner, M.: Combinatorial theory. Springer, New York, Berlin (1979)

Bass, H.: Algebraic K-theory. W. A. Benjamin Inc, New York, Amsterdam (1968)

Bernstein, J., Frenkel, I., Khovanov, M.: A categorification of the Temperley-Lieb algebra and Schur quotients of $U\left(\mathfrak{s l}_{2}\right)$ via projective and Zuckerman functors. Selecta Math. (N.S.) 5(2), 199-241 (1999)

Chuang, J., Rouquier, R.: Derived equivalences for symmetric groups and $\mathfrak{s l}_{2}$-categorification. Ann. Math. 167(1), 245-298 (2008)

Elias, B.: Soergel diagrammatics for dihedral groups. PhD thesis, Columbia University (2011)

Golan, J.: Semirings and their applications. Kluwer Academic Publishers, Dordrecht (1999)

Grensing, A.-L., Mazorchuk, V.: Categorification using dual projection functors. arXiv:1501.00095 (2014)

Grensing, A.-L., Mazorchuk, V.: Categorification of the Catalan monoid. Semigroup Forum 89(1), 155-168 (2014)

Guo, X., Xi, Ch.: Cellularity of twisted semigroup algebras. J. Pure Appl. Algebra 213(1), 71-86 (2009)

Hebisch, U.: Eine algebraische Theorie unendlicher Summen mit Anwendungen auf Halbgruppen und Halbringe. Bayreuth. Math. Schr. 40, 21-152 (1992)

Johnson, J., Manes, E.: On modules over a semiring. J. Algebra 15, 57-67 (1970)

Karner, G.: On limits in complete semirings. Semigroup Forum 45(2), 148-165 (1992)

Kazhdan, D., Lusztig, G.: Representations of Coxeter groups and Hecke algebras. Invent. Math. 53(2), 165-184 (1979)

Khovanov, M.: A categorification of the Jones polynomial. Duke Math. J. 101(3), 359-426 (2000)

Kudryavtseva, G., Mazorchuk, V.: On multisemigroups. Portugal. Math. (N.S.) 72(1), 47-80 (2015)

Leinster, T.: Basic bicategories. Preprint arXiv:math/9810017 (1998)

Martin, P., Mazorchuk, V.: Partitioned binary relations. Math. Scand. 113(1), 30-52 (2013)

Mazorchuk, V.: Lectures on algebraic categorification. European Mathematical Society (EMS), Zürich, QGM Master Class Series (2012)

Mazorchuk, V., Miemietz, V.: Cell 2-representations of finitary 2-categories. Compos. Math. 147, 15191545 (2011)

Mazorchuk, V., Miemietz, V.: Additive versus abelian 2-representations of fiat 2-categories. Moscow Math. J. 14(3), 595-615 (2014)

Mazorchuk, V., Miemietz, V.: Morita theory for finitary 2-categories. Quantum Topol. 7(1), 1-28 (2016a)

Mazorchuk, V., Miemietz, V.: Endomorphisms of cell 2-representations. IMRN (2016b) (to appear)

Mitchell, B.: Rings with several objects. Adv. Math. 8, 1-161 (1972)

Soergel, W.: Kazhdan-Lusztig-Polynome und unzerlegbare Bimoduln über Polynomringen. J. Inst. Math. Jussieu 6(3), 501-525 (2007)

Wilcox, S.: Cellularity of diagram algebras as twisted semigroup algebras. J. Algebra 309(1), 10-31 (2007)

Zhang, X.: Duflo involutions for 2-categories associated to tree quivers. J. Algebra Appl. 15(3), 1650041 (2015a). doi:10.1142/S0219498816500419

Zhang, X.: Simple transitive 2-representations and Drinfeld center for some finitary 2-categories. Preprint arXiv:1506.02402 (2015b) 